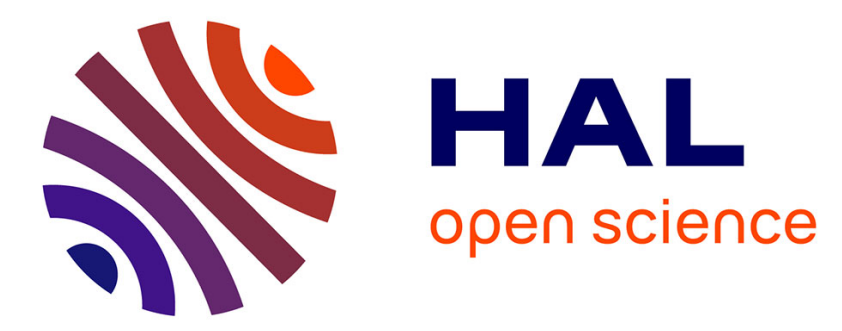

\title{
Electron acceleration by surface plasma waves in the interaction between femtosecond laser pulses and sharp-edged overdense plasmas
}

J Kupersztych, M Raynaud, C Riconda

\section{- To cite this version:}

J Kupersztych, M Raynaud, C Riconda. Electron acceleration by surface plasma waves in the interaction between femtosecond laser pulses and sharp-edged overdense plasmas. Physics of Plasmas, 2004, 10.1063/1.1650353 . hal-01327445

\section{HAL Id: hal-01327445 \\ https://hal.science/hal-01327445}

Submitted on 8 Jun 2016

HAL is a multi-disciplinary open access archive for the deposit and dissemination of scientific research documents, whether they are published or not. The documents may come from teaching and research institutions in France or abroad, or from public or private research centers.
L'archive ouverte pluridisciplinaire HAL, est destinée au dépôt et à la diffusion de documents scientifiques de niveau recherche, publiés ou non, émanant des établissements d'enseignement et de recherche français ou étrangers, des laboratoires publics ou privés. 


\title{
Electron acceleration by surface plasma waves in the interaction between femtosecond laser pulses and sharp-edged overdense plasmas
}

\author{
J. Kupersztych, M. Raynaud, and C. Riconda ${ }^{\text {a) }}$ \\ DSM/DRECAM/SPCSI, CEA/Saclay, 91191 Gif-sur-Yvette Cedex, France
}

(Received 25 August 2003; accepted 28 December 2003; published online 2 April 2004)

\begin{abstract}
The relativistic acceleration of electrons by the field of surface plasma waves created in the interaction between ultrashort high-intensity laser pulses with sharp-edged overdense plasmas has been investigated. It is shown that the initial phase of the wave experienced by the electrons play a leading part by yielding a well-defined peaked structure in the energy distribution function. This study suggests that resonant excitation of surface plasma waves could result in quasi-monokinetic energetic electron bunches. When the space charge field becomes too strong, this mechanism can evolve toward a true absorption process of the surface wave energy via an enhanced "vacuum heating" mechanism generalized to the case of surface plasma waves. (C) 2004 American Institute of Physics. [DOI: 10.1063/1.1650353]
\end{abstract}

\section{INTRODUCTION}

It is well known that electrons trapped in traveling plasma waves can be accelerated to high energies. ${ }^{1}$ Also, the proposal of direct excitation of plasma waves by lasers has greatly stimulated theoretical and experimental investigations toward the development of plasma-based electron accelerators. Among various acceleration schemes, ${ }^{2,3}$ particular attention has been focussed on the possibility of creating large amplitude plasma waves in the wake of a laser pulse. ${ }^{4}$ Recently, with the arrival of laser systems capable of delivering light pulses of extremely high intensities (of the order of $10^{21} \mathrm{~W} \mathrm{~cm}^{-2}$ ) in the femtosecond regime and with high temporal contrast (better than $\left.10^{8}\right),{ }^{5}$ much attention has been raised to absorption mechanisms and high energy electron generation involving overdense plasmas with stepwise density profiles resulting from laser-solid interaction. ${ }^{6-8}$

When an intense ultrashort (say $100 \mathrm{fs}$ ) laser pulse is impinging onto a solid target, multiple ionization rapidly occurs without significant ablation. The hydrodynamic expansion, partially reduced by the radiative pressure of the laser, has not enough time to smooth the density gradient $\left[\mathrm{d}\left(\ln n_{i}\right) / \mathrm{d} x \gg \lambda_{0}^{-1}\right.$, where $n_{i}$ denotes the ion density and $\lambda_{0}$ the laser wavelength] and an overdense plasma can thus be created which can keep the steepened density profile of the original target. The electromagnetic energy is then absorbed in an optical skin depth by collisional processes and by collisionless mechanisms such as sheath inverse Bremsstrahlung, ${ }^{9} \vec{J} \times \vec{B}$ heating, ${ }^{10}$ vacuum heating ${ }^{11}$ and anomalous skin-layer heating, ${ }^{12}$ while the electron thermal energy is correlatively transported into the dense cold plasma.

To improve conversion of the laser energy into electron energy, advantage can be taken of some peculiar features of these plasmas. In particular, the boundary between the overdense plasma and the vacuum (or a low-density plasma) can

a) Also at Istituto Nazionale Fisica della Materia, Sez.A Udr. Pisa, Italy. support surface plasma waves. ${ }^{13-16}$ Thus, under certain conditions, a laser can resonantly excite a surface plasma wave localized at the plasma-vacuum interface and characterized by a large electric field perpendicular to the surface. This field can then strongly accelerate electrons toward the vacuum more efficiently than the laser. It is worth noting that resonant excitation of surface plasma waves has been recently experimentally realized by using a structured metal surface, such as a periodic grating. It has been shown that, in the framework of multiphoton photoelectric emission of metals, the laser excitation of surface plasma waves can lead to considerable modification of the electron energy spectrum and increasing of the laser absorption, together with enhanced electron production. ${ }^{17}$ In this context, energetic photoelectrons have been produced with energies up to $400 \mathrm{eV}$ for a laser intensity of $10^{13} \mathrm{~W} / \mathrm{cm}^{2} .{ }^{18}$

The aim of this paper is to show that, in the scheme that consists in resonantly exciting surface plasma waves at the surface of a sharp-edged overdense plasma by ultrashort high-intensity laser pulses (for which relativistic effects need to be taken into account), very energetic electron bunches can be produced with interesting characteristics. This regime which includes modifications in the conditions of excitation of the surface wave as well as in the energy gain by the electrons, has not yet been fully explored theoretically nor experimentally. Furthermore, it is worth pointing out that, depending on the actual experimental situation, these energetic electrons produced during the interaction may deposit their energy back into the plasma due to the occurrence of strong space charge fields. This process may thus also be considered as a new absorption mechanism of the laser pulse energy by the plasma in ultrashort time scales.

\section{ELECTRON ACCELERATION BY SURFACE PLASMA WAVES (NONRELATIVISTIC CASE)}

We consider a sharp-edged overdense plasma resulting from the multiple ionization of a metal target consisting of a grating. Such a structured surface makes it possible to reso- 
nantly excite surface plasma waves by a $p$-polarized laser wave. As a matter of fact, as the phase velocity of the surface plasma waves is lower than the light velocity, additional device is required for momentum conservation. This can be conveniently obtained by means of a periodically engraved metal surface, that is, a grating. ${ }^{19}$ This periodic structure provides the increment to the light wave momentum to reach the momentum value of the surface wave as a consequence of Bloch's theorem. Thus, if a $p$-polarized laser wave of angular frequency $\omega_{0}$ is impinging onto the metal surface with an angle $\vartheta$, the component $k_{0 \|}$ of the photon wave vector along the periodic surface can be expressed as $k_{0 \|}=\left(\omega_{0} / c\right)(\sin \vartheta$ $+\lambda_{0} / a$ ), where $a$ denotes the grating period. The energy and momentum conservation equations in "photon-plasmon" scattering can thus be written as $\omega=\omega_{0}$ and $k_{\|}=k_{0 \|}$, where $\omega$ and $k_{\|}$, respectively, denote the frequency and wave vector of the surface plasma wave directed along the direction of the metal-vacuum interface. Describing the plasma as an electron fluid neutralized by uniform background and considering that the thermal corrections are negligible in this range of temperatures, the dispersion relation takes the form ${ }^{13}$

$$
k_{\|}^{2} c^{2} / \omega^{2}=\left(\omega^{2}-\omega_{p e}^{2}\right) /\left(2 \omega^{2}-\omega_{p e}^{2}\right),
$$

where $\omega_{p e}$ denotes the plasma frequency. The field of the laser-resonantly excited surface waves decays toward the vacuum over the characteristic distance (evanescence length), ${ }^{13} L_{E}=\left(k_{\|}^{2}-\omega^{2} / c^{2}\right)^{-1 / 2}$. The maximum amplitude $E_{s w}$ of the surface wave field is related to the laser field amplitude $E_{0}$ by the expression $E_{s w}=\eta E_{0}$ where $\eta$ denotes the resonance factor. An estimate of this factor can be obtained in the framework of the following simple model. We shall assume that the amount of electromagnetic energy stocked in the resonant plasma wave, which can be estimated as $S L_{E} E_{s w}^{2} / 8 \pi$ (where $S$ denotes the laser focal spot), results from conversion of the electromagnetic energy absorbed by the plasma during the laser pulse duration $\tau_{L}$. If $\alpha$ denotes the absorption rate, this quantity can be estimated as $\alpha c \tau_{L} S E_{0}^{2} / 4 \pi$. Conservation of energy thus leads to the expression $\eta^{2}=2 \alpha c \tau_{L} / L_{E}$. For example, for a laser wavelength $\lambda_{0}=1.06 \mu \mathrm{m}$, a pulse duration $\tau_{L}=30 \mathrm{fs}$, and a plasma density $n_{e}=25 n_{c}=2.47 \times 10^{22} \mathrm{~cm}^{-3}$ where $n_{c}$ $=4 \pi e^{2} / m \omega_{0}^{2}$, we find $L_{E}=0.8 \mu \mathrm{m}$. If we assume an absorption rate $\alpha$ of only $50 \%$, the resonant factor can be estimated as $\eta^{2} \sim 10$. In this model, the energy transferred to the hot electron population during the ultrashort growing time of the resonance is neglected, so that the electron acceleration process is assumed to begin at the optimum of the resonance (that is, when $\eta^{2} \sim 10$ ).

The electric field of the resonant surface plasma wave in the vacuum can be expressed as

$$
\begin{aligned}
E_{x}= & E_{s w} \exp \left(-x / L_{E}\right) \exp \left(-t^{2} / 2 \tau_{s w}^{2}\right) \cos \left(\omega_{0} t+\phi\right. \\
& +\pi / 2),
\end{aligned}
$$

where $x$ is the coordinate along the normal to the surface, $\phi$ is the phase and $\tau_{s w}$ denotes the effective wave lifetime. In the following, the component $E_{\|}$of the field parallel to the surface is neglected as it is much smaller than $E_{x} \cdot{ }^{13}$ It is instructive to consider first the nonrelativistic case corre- sponding to moderate laser intensities. The behavior of an electron entering at the plasma surface in the evanescent electric field of the surface plasma wave is characterized by the following two parameters: its quiver velocity $v_{\text {osc }}$ $\equiv e E_{s w} / m \omega_{0}$ and a typical length, $\Lambda_{\text {osc }} \equiv v_{\text {osc }} / \omega_{0}$, representative of its high frequency motion in the vacuum dressed by the high-amplitude oscillating field. If the condition $L_{E} / \Lambda_{\text {osc }} \gg 1$ is satisfied, electrons will have enough time to perform several oscillations before leaving the surface wave field. As a consequence, they will experience the effect of the ponderomotive force due to this strongly inhomogeneous field, the role of which is to convert the electron quiver energy into net kinetic energy. In this case, the typical kinetic energy that can be acquired by an electron is equal to the ponderomotive potential $U_{\mathrm{osc}}=(1 / 4) m v_{\mathrm{osc}}^{2}$, provided the coupling with the field is adiabatically turned off.

Actually, strong spatial inhomogeneity is not the only source of nonlinearity in this problem. The electrons that reach the surface are suddenly coupled to the external field at the moment when they enter into the surface plasma wave. ${ }^{17}$ They can therefore be accelerated during less than a period of the wave before even feeling the effect of the ponderomotive force. This extra kinetic energy may be simply related to the motion of an electron entering at a given moment of time when the phase is $\phi$ in a spatially constant oscillatory field. In the nonrelativistic case, the resulting electron velocity can be simply expressed as $\langle v(\phi)\rangle_{\mathrm{hf}}=v_{0}-v_{\text {osc }} \cos \phi$, where it can be assumed that its initial velocity $v_{0} \sim v_{\text {the }}$. The electron final kinetic energy $\mathcal{E}$, in the case of full conversion of the ponderomotive potential, ${ }^{17}$ is therefore $\mathcal{E}=U_{\text {osc }}$ $+2 U_{\text {osc }}\left(v_{0} / v_{\text {osc }}-\cos \phi\right)^{2}$, whence it is seen that those electrons that have experienced the best phase $(\phi=\pi)$ are accelerated to energies higher than the ponderomotive potential $U_{\text {osc }}$.

\section{ELECTRON ACCELERATION BY SURFACE PLASMA WAVES (RELATIVISTIC CASE)}

Let us consider still higher laser intensities such that the electron motion becomes relativistic. The quiver velocity is now $v_{\text {osc }}=p_{\text {osc }} / m \gamma_{\text {osc }}$, with $\gamma_{\text {osc }}=\left(1+p_{\text {osc }}^{2} / m^{2} c^{2}\right)^{1 / 2}$ and $p_{\text {osc }} \equiv e E_{s w} / \omega_{0}=8.6 \times 10^{-10}\left[\eta^{2} I_{0}\left(\mathrm{~W} / \mathrm{cm}^{2}\right) \lambda_{0}^{2}\left(\mu \mathrm{m}^{2}\right)\right]^{1 / 2}$. If we consider the value of the laser parameter $I_{0} \lambda_{0}^{2}$ $=10^{19} \mathrm{~W} \mu \mathrm{m}^{2} / \mathrm{cm}^{2}$, we are well into the relativistic regime with $v_{\text {osc }} \sim c$ and $\gamma_{\text {osc }}=8.7$. In this case, the relativistic problem reveals basic differences with respect to the nonrelativistic limit, as we shall show now.

Relativistic corrections to the momentum of an electron in a relativistic wave lead to modifications in the corresponding dispersion relation. For example, in the case of a circularly polarized electromagnetic wave traveling in a plasma, the well-known exact dispersion relation ${ }^{20}$ is $\omega_{0}^{2}=\omega_{p e}^{2} / \gamma_{\text {osc }}$ $+k_{0}^{2} c^{2}$, where $\gamma_{\text {osc }}$ is the relevant relativistic factor. Recent numerical simulations ${ }^{16}$ of surface plasma waves in relativistic regime are consistent with a relativistically modified dispersion relation (1), where $\omega_{p e}^{2}$ is analogously replaced by $\omega_{p e}^{2} / \gamma_{\text {eff }}$, with $\gamma_{\text {eff }}=\left(1+\left\langle p_{\text {osc }}^{2}\right\rangle_{\text {av }} / m^{2} c^{2}\right)^{1 / 2}=\left(1+p_{\text {osc }}^{2} /\right.$ $\left.2 m^{2} c^{2}\right)^{1 / 2}$. It follows that the field amplitude can be saturated as a consequence of the relativistic detuning that occurs 


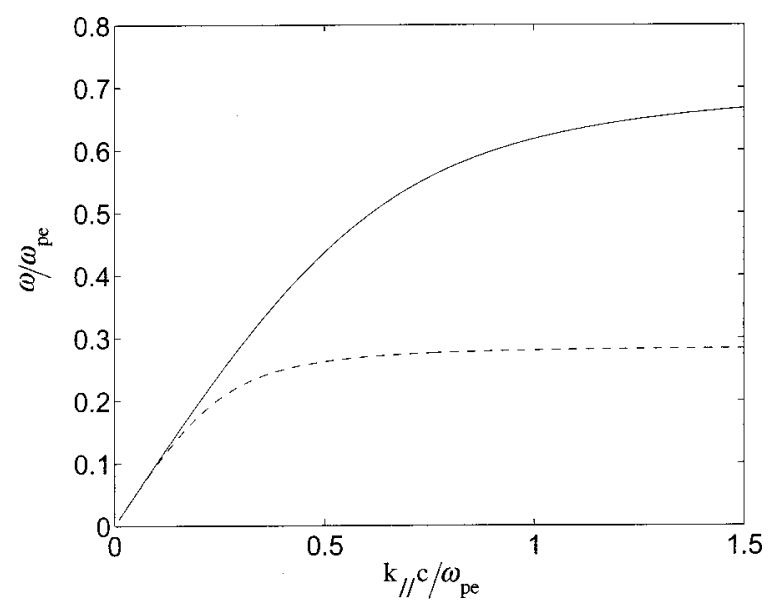

FIG. 1. Dispersion relation (1) for surface waves (plain line) and its relativistic generalization for $\gamma_{\mathrm{eff}}=6.2$ (dotted line).

while the resonance grows in time. However, as it is shown in Fig. 1, this resonance detuning can be restricted to acceptable values by choosing conveniently the laser frequency in order to satisfy the following condition: $\omega_{0} \ll \omega_{p e}$. For example, if we assume $\omega / \omega_{p e}=0.2$, the relativistic detuning is less than $\delta \omega / \omega \sim 10 \%$, a value which is just of the order of magnitude of the laser spectrum width for a laser pulse duration of $30 \mathrm{fs}$. Hence, to overcome the relativistic detuning obstacle, we shall assume the condition $\omega_{0} \ll \omega_{p e}$, which corresponds to a regime where the scale of variation of the field is greater than or of the same order as the distance explored by the electrons in the wave, $L_{E} \geqslant \Lambda_{\text {osc }}$. In this regime, the electrons will oscillate in the evanescent field of the relativistic surface wave, feeling the effect of their initial phase together with the effect of the field intensity gradient force.

\section{NUMERICAL STUDY OF ELECTRON ACCELERATION BY RELATIVISTIC SURFACE PLASMA WAVES}

Taking advantage of our simplified approach, we performed a systematic study of the nonlinear relativistic electron behavior in the above one-dimensional surface wave field $E_{x}$ by using a 1D-test particle numerical simulation that has enabled us to identify the most relevant set of parameters. Such a numerical approach is appropriate to the characteristics of this problem, namely the very small Debye length $\left(\lambda_{D} \sim 10^{-4}-10^{-3} \lambda_{0}\right)$ due to the assumed high electron density and relatively cold bulk electron temperature. It is worth pointing out that, for the taken values of plasma temperature and electron density to critical density ratio (i.e., $k \lambda_{D} \ll 1$ ), the possible coupling of large-amplitude surface plasma waves with other collective oscillations such as surface oscillations at $2 \omega$ (Ref. 21) and ion plasma waves induced by frustrated Debye shielding ${ }^{22}$ is minimized and is therefore neglected in the following.

In our simulation, the overdense plasma thus results from multiple ionization of a metallic target by a $p$-polarized laser pulse of duration $\tau_{L} \sim 30 \mathrm{fs}$. A good candidate can be an aluminum target yielding helium-like ions $(Z=11)$. The

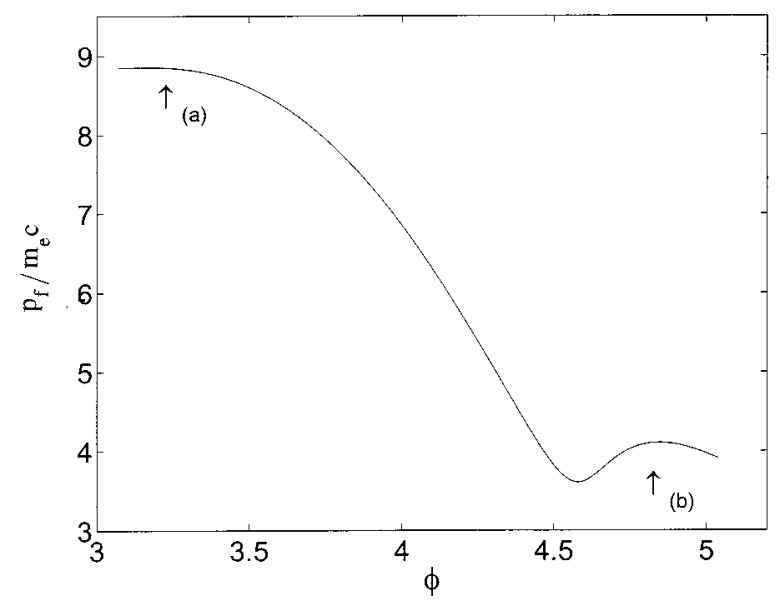

FIG. 2. Electron final momentum $p_{f} / m c$ as a function of the entry phase $\phi$ in the field of the surface wave.

bulk electron temperature is assumed $T_{e}=0.1-1 \mathrm{keV}$ and its density $n_{e}$ so that $n_{e} \sim 25, n_{c}=2.47 \times 10^{22} \mathrm{~cm}^{-3}{ }^{6}$ The laser wavelength is $\lambda_{0}=1.06 \mu \mathrm{m}$ and the relevant laser parameter is taken to be $I_{0} \lambda_{0}^{2}=10^{19} \mathrm{~W} \mu \mathrm{m}^{2} / \mathrm{cm}^{2}$. A surface plasma wave of frequency $\omega=0.2 \omega_{p e}$ is then resonantly excited during the laser pulse with a resonant factor $\eta^{2}=10$. The peak amplitude of its field is therefore $\eta^{2} I_{0} \lambda_{0}^{2}$ $=10^{20} \mathrm{~W} \mu \mathrm{m}^{2} / \mathrm{cm}^{2}$ and its evanescence length is $L_{E}$ $=0.8 \mu \mathrm{m}$. We are interested in considering the first stage of the interaction between the surface plasma wave and the electrons. The lapse of time $\tau_{\text {int }}$ during which such an interaction is possible can be estimated by expressing the condition that the evanescence length $L_{E}$ of the surface plasma wave is larger than the plasma expansion length $L_{\text {exp }}$. An overestimate of this last quantity can be obtained as $L_{\exp }$ $\sim V_{s} \tau_{\text {int }}$, where we have introduced an effective ion-acoustic velocity $V_{s}=\sqrt{Z W_{\text {eff }} / m_{i}}$, where $W_{\text {eff }}=\left(\gamma_{\text {eff }}-1\right) m c^{2}$ is the effective ponderomotive energy of the electrons in the field of the surface plasma wave at its peak amplitude. Hence, for the above values of the relevant parameters, the condition $L_{\text {exp }} / L_{E} \ll 1$ is satisfied for $\tau_{\text {int }} \sim 10$ fs.

In the computations, the relativistic equations of motion were solved with the leapfrog method. We have considered electrons loaded in a buffer with a given momentum distribution function, introduced as a flux coming from inside the plasma. At the plasma-vacuum interface, the electrons enter into the field of the wave at random times (which is equivalent to electrons seeing different phases $\phi$ ) over the whole interaction time $\tau_{\text {int }}=10$ fs. Thus, electrons entering into the field over time intervals of the order of $\tau_{\text {int }}$ will experience various values of the maximum envelope amplitude, leading to different values of $p_{\text {osc }}$. The final momentum values are recorded once the particles have left the field.

To analyze the electron motion in details, we have first investigated a situation where the particles have entered into the field during only one period at the envelope peak $(t$ $=0$ ), that is, for the maximum intensity of the resonant field. The resulting electron final momentum as a function of the entry phase is reported in Fig. 2. This figure exhibits the existence of three extrema in the momentum space: a very 


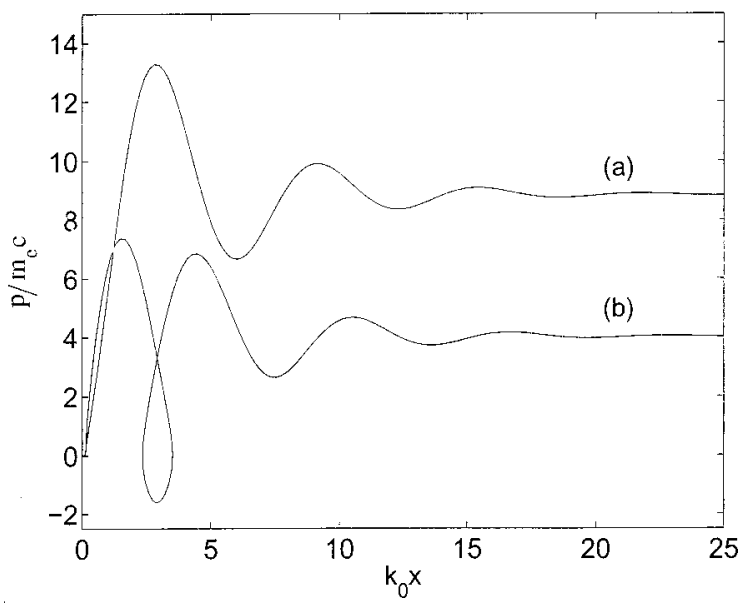

FIG. 3. Single electron orbits in phase space for the two entry phases $\phi$ $=\pi$ (a) and $\phi=3 \pi / 2$ (b), corresponding to the two maxima shown in Fig.

flat maximum around the "best" value $\phi=\pi$ of the phase, a minimum and another maximum in a region near the less favorable phase $(\phi=3 \pi / 2)$. The existence of an extremum means that many particles seeing different phases will have the same momentum value, resulting in a bunching of the electrons in momentum space, and in the occurence of a well-defined peak in the energy spectrum. Thus, the wide "plateau" near $\phi=\pi$ [labeled (a)] shows that a large number of electrons will reach the maximum kinetic energy $\mathcal{E}_{\max }$ $\sim m c^{2}\left(p_{f \max } / m c-1\right) \sim m c^{2}\left(\gamma_{\mathrm{osc}}-1\right) \sim 4 \mathrm{MeV}$ while the lower "plateau" near $\phi=3 \pi / 2$ [labeled (b)] corresponds to a smaller number of electrons that have acquired $\sim 1.5 \mathrm{MeV}$. The trajectories associated with these two "plateaus" are depicted in Fig. 3. The orbit labeled (b) shows that the electron that has experienced the "worst" initial phase, after being accelerated in the wave, is bounced back. The time spent while bouncing back is such that it acts for the electron as a sort of resetting of the initial condition, so that the particle can experience the most favorable phase after the turn and gain enough energy to move away from the high field region. Hence, the "worst" initial phase does not necessarily correspond to the ponderomotive potential as it was the case in the nonrelativistic regime. This is related to the fact that the concept of ponderomotive potential, which is based on a net separation between high and low frequency motions together with a linearization about the spatial variation of the field with respect to its intensity, ${ }^{23}$ requires here the condition $\Lambda_{\text {osc }} \ll L_{E}$, which is not satisfied in the present relativistic case.

In the computation of the electron distribution function, we have taken into account the fact that the particles enter into the field during the whole interaction time $\left(0<t<\tau_{\text {int }}\right)$ with the surface wave. The resulting electron energy distribution is plotted in Fig. 4 with a resolution of $\delta E=6$ $\times 10^{-3} \mathrm{MeV}$ and presents a well-defined peaked structure. The form of the distribution function can be easily understood in terms of the above discussion relative to Figs. 2 and 3 that apply to the case of electrons entering in the field during one period only. Thus, the multipeaked distribution

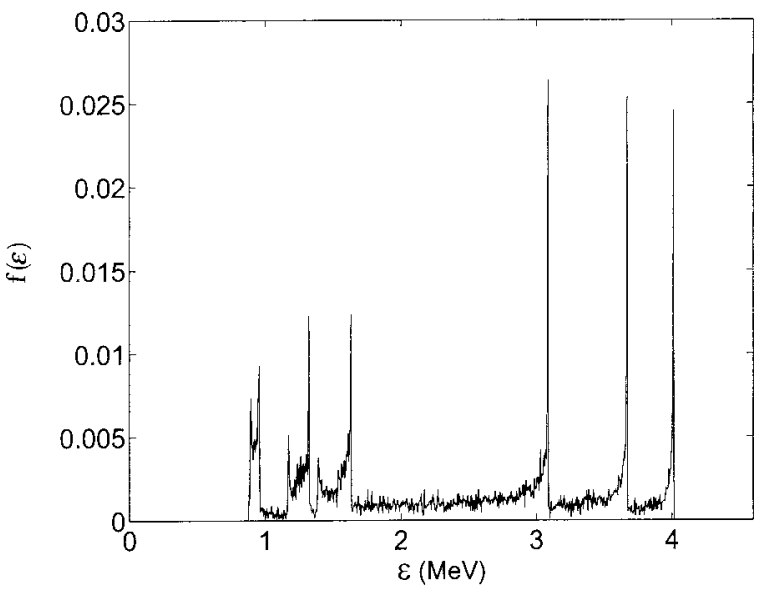

FIG. 4. The "bunched" electron distribution function $f(\mathcal{E})$.

function is the macroscopical signature of phase bunching effects corresponding to those electrons that have seen the most favorable phases of the field during the whole interaction time $\tau_{\text {int }}$. The number of peaks is therefore directly related to the number of cycles of the wave during the interaction time (i.e., three cycles during $10 \mathrm{fs}$ ). Furthermore, another consequence of the phase effects is that this emission of quasi-monokinetic energetic electron bunches is pulsed with frequency $\omega_{0}$, as confirmed by the phase space. Electron bunches have also been considered in other electron acceleration schemes and have been the subject of specific experimental and numerical investigations. ${ }^{24}$

\section{CONCLUSION}

We have shown that resonant excitation of surface plasma waves by ultrashort high-intensity laser pulses can produce quasi-monokinetic relativistic electron bunches. Let us mention that generation of ultrashort electron bunches is of interest for producing ultrashort radiation sources in the $\mathrm{x}$-ray range, for example, or for radiation-based diagnostics. $^{25}$

Some consequences of the mechanism discussed here can be prospected owing to its characteristics, namely ultrashort interaction time scales together with strongly evanescent fields. Thus, it can be expected that the charge separation field that could develop at the plasma surface as the electron emission increases would play an important part only after several cycles, that is, after significant emission of electron bunches has occurred. Then, depending on the nature of the target, ${ }^{26}$ the presence and setup of strong space charge fields will govern the subsequent evolution of the hot electron population, either dragging the ions out of the plasma $^{27}$ or retaining the electron bunches and recoiling them back in the neutralizing plasma. In this last situation, however, we shall deal with a true conversion process of the surface wave energy, in a form that can be considered as an enhanced "vacuum heating," 11 generalized to the case of surface plasma waves resonantly excited by laser. 


\section{ACKNOWLEDGMENTS}

Valuable discussions with F. Amiranoff, O. Gobert, and F. Pegoraro are gratefully acknowledged.

${ }^{1}$ J. M. Dawson, Phys. Rev. 113, 383 (1959).

${ }^{2}$ Laser Acceleration of Particles, edited by P. J. Channell (AIP, New York, 1982) and references therein; E. Esarey, P. Sprangle, J. Krall, and A. Ting, IEEE Trans. Plasma Sci. 24, 252 (1996).

${ }^{3}$ The concept of a plasma grating accelerator was discussed in Laser Acceleration of Particles, edited by C. Joshi and T. C. Katsouleas (AIP, New York, 1985).

${ }^{4}$ T. Tajima and J. M. Dawson, Phys. Rev. Lett. 43, 267 (1979); C. Joshi, W. B. Mori, T. Katsouleas et al., Nature (London) 311, 525 (1984); T. C. Chiou, T. Katsouleas, C. Decker et al., Phys. Plasmas 2, 310 (1995); D. Umstadter, J. K. Kim, and E. Dodd, Phys. Rev. Lett. 76, 2073 (1996); E. Esarey, R. F. Hubbard, W. P. Leemans et al., ibid. 79, 2682 (1997); V. Yakimenko, I. V. Pogorelsky, I. V. Pavlishin et al., ibid. 91, 014802 (2003); N. Saito and A. Ogata, Phys. Plasmas 10, 3358 (2003); L. M. Gorbunov, P. Mora, and R. R. Ramazashvili, ibid. 10, 4563 (2003).

${ }^{5}$ G. Chériaux, P. D’Olivera, G. Rey et al., Proceedings of C.L.E.O. 2002 Conference, Long Beach, CA (Optical Society of America, Washington, DC, 2002), paper CMK2.

${ }^{6}$ H. M. Milchberg, R. R. Freeman, and S. C. Davey, Phys. Rev. Lett. 61, 2364 (1988); M. M. Murnane, H. C. Kapteyn, and R. W. Falcone, ibid. 62, 155 (1989); J. A. Cobble, G. A. Kyrala, A. A. Hauer et al., Phys. Rev. A 39, 454 (1989); J. C. Gauthier, J. P. Geindre, P. Audebert et al., Phys. Plasmas 4, 1811 (1997); K. Eidmann, R. Rix, T. Schlegel, and K. Witte, Europhys. Lett. 55, 334 (2001).

${ }^{7}$ M. I. K. Santala, M. Zepf, I. Watts et al., Phys. Rev. Lett. 84, 1459 (2000); X. Wang, M. Krishnan, N. Saleh et al., ibid. 84, 5324 (2000); W. Yu, V. Bychenkov, M. Y. Sentoku et al., ibid. 85, 570 (2000); Q. L. Dong and J. Zhang, Phys. Plasmas 8, 1025 (2001).
${ }^{8}$ J. Kupersztych and M. Raynaud, Phys. Rev. E 59, 4559 (1999) and references herein.

${ }^{9}$ P. J. Catto and R. M. More, Phys. Fluids 20, 704 (1977).

${ }^{10}$ W. Kruer and K. Estabrook, Phys. Fluids 28, 430 (1985).

${ }^{11}$ F. Brunel, Phys. Rev. Lett. 59, 52 (1987).

${ }^{12}$ S. Ichimaru, Basic Principles of Plasma Physics (Benjamin, Reading, MA, 1973).

${ }^{13}$ P. K. Kaw and J. B. McBride, Phys. Fluids 13, 1784 (1970).

${ }^{14}$ J. M. Kindel, K. Lee, and E. L. Lindman, Phys. Rev. Lett. 34, 134 (1975).

${ }^{15}$ R. Dragila and P. Vukovic, Phys. Rev. Lett. 61, 2759 (1988).

${ }^{16}$ A. Macchi, F. Cornolti, F. Pegoraro et al., Phys. Rev. Lett. 87, 205004 (2001).

${ }^{17}$ J. Kupersztych, P. Monchicourt, and M. Raynaud, Phys. Rev. Lett. 86, 5180 (2001).

${ }^{18}$ J. Zawadzka, D. A. Jaroszynski, J. J. Carey, and K. Wynne, Nucl. Instrum. Methods Phys. Res. A 445, 324 (2000); Appl. Phys. Lett. 79, 2130 (2001).

${ }^{19}$ H. Raether, Surface Plasmons on Smooth and Rough Surfaces and Gratings (Springer-Verlag, Berlin, 1988).

${ }^{20}$ A. I. Akhiezer and R. V. Polovin, Sov. Phys. JETP 5, 696 (1956).

${ }^{21}$ R. Lichters, J. Meyer-ter-Vehn, and A. Pukhov, Phys. Plasmas 3, 3425 (1996).

${ }^{22}$ R. P. Drake and R. S. Marjoribanks, Phys. Plasmas 9, 267 (2002).

${ }^{23}$ J. Kupersztych, Phys. Rev. Lett. 54, 1385 (1985).

${ }^{24}$ E. Esarey, C. B. Schroeder, W. P. Leemans, and B. Hafizi, Phys. Plasmas 6, 2362 (1999); D. Giulietti, M. Galimberti, A. Giulietti et al., ibid. 9, 3655 (2002); S. D. Baton, J. J. Santos, F. Amiranoff et al., Phys. Rev. Lett. 91, 105001 (2003)

${ }^{25}$ P. P. Rajeev, P. Taneja, P. Ayyub et al., Phys. Rev. Lett. 90, 115002 (2003); W. P. Leemans, C. G. R. Geddes, J. Faure et al., ibid. 91, 074802 (2003).

${ }^{26}$ R. Stoian, A. Rosenfeld, D. Ashkenasi et al., Phys. Rev. Lett. 88, 097603 (2002).

${ }^{27}$ M. Zepf, E. L. Clark, K. Krushelmich et al., Phys. Plasmas 8, 2323 (2001) 